\title{
Application of differential quadrature method to investigate dynamics of a curved beam structure acted upon by a moving concentrated load
}

\author{
Ali Nikkhoo ${ }^{1}$, Hassan Kananipour ${ }^{*}{ }^{1}$, Hossein Chavoshi ${ }^{1}$ and Raham Zarfam ${ }^{2}$ \\ ${ }^{1}$ Department of Civil Engineering, University of Science and Culture, Tehran, Iran. \\ ${ }^{2}$ Departments of Civil Engineering, Sharif University of Technology, Tehran, Iran. \\ h.kananipour@usc.ac.ir, nikkhoo@usc.ac.ir, h.chavoshi@ymail.com, r_zarfam@civil.sharif.edu,
}

\begin{abstract}
Application of curved beams in special structures requires a special analysis. In this study, the differential quadrature method (DQM) as a well-known numerical method is utilized in the dynamic analysis of the Euler-Bernoulli curved beam problem with a uniform cross section under a constant moving load. DQ approximation of the required partial derivatives is given by a weighted linear sum of the function values at all grid points. A prismatic semicircular arch with simply supported boundary conditions is assumed. The accuracy of the obtained results is corroborated by employing the Galerkin and finite element methods. Finally, the convergence rate of the DQM and Finite Element Method (FEM) in the associated problem is explored. In the structural problems with specific geometry, use of DQM which is independent of domain discretization, is proved to be efficient.
\end{abstract}

Keywords: Differential Quadrature Method (DQM), Semicircular curved beam, Moving load, Galerkin method, Finite element method.

\section{Introduction}

Differential Quadrature Method (DQM) is a proper method in solving mathematic and engineering equations straightly. This method estimates a partial derivative of a variable function with respect to a coordinate at a grid point as a weighted linear sum of the function values at all grid points along that coordinate direction. DQ method was introduced for the first time by Bellman and Casti (1971), and was applied in solving partial differential equations by Bellman et al. (1972). Bert et al. (1988) and Jang et al. (1989) utilized DQ method in structural mechanic problems for the first time. However, DQ method application in continuum mechanics is extremely restricted to those problems with a regular domain and specific boundary conditions. One of the DQ method applications in solving beam problems was studied by Laura and Gutierrez (1993) in Timoshenko beam free vibration with the classical boundary conditions. Liu and Wu (2001) have conducted research on free vibration of Euler-Bernoulli beam employing this method.

DQ method application in solving dynamic equations is difficult to cope with due to the simultaneous existence of boundary and initial conditions. Analysis of structural problems via this method is frequently carried out to static analysis. In this paper, DQM application will be investigated grounded on Euler-Bernoulli beam theory, which is appropriate for analysis of beams limited in size and also multi-span beams. Initially, essentials and the equations dominating DQM will be explained. Subsequently, dynamic equations governing the curved beam will be extracted, and DQM will be applied in the mentioned beam analysis with simply supported (SS-SS) boundary conditions. Finally, it will be illustrated that DQM is applicable in the curved beams dynamic analysis excited by a moving load, and its convergence will be explored.

\section{Differential quadrature method (DQM)}

Differential Quadrature (DQ) is capable of calculating derivative orders of the field variable up to $\mathrm{N}-1$ order in the case of $N$ grid points. $D Q$ equations based on polynomial or Fourier series expansion are computable; in this paper, $D Q$ based on polynomials, which provides fine compatibility in analyzing high-order differential equations, is employed. A test function is required for deriving DQ equations; moreover, Shu (2000) proved the Lagrange interpolation polynomials as the test function generates the best convergence. The value derived for the function $w(x)$ in the th grid point is calculated via summing weighting-linear function values in the other nodes (Eq. (1)). Besides, $n$ th-order derivative $(n=2,3, \ldots$, $N-1)$ in the th grid point can be calculated in the same way(Eq. (2))

$$
\begin{aligned}
& \frac{d w\left(x_{i}\right)}{d x}=\sum_{k=1}^{N} c_{i k}^{(1)} w\left(x_{k}\right), \\
& \frac{d^{n} w\left(x_{i}\right)}{d x^{n}}=\sum_{k=1}^{N} c_{i k}^{(n)} w\left(x_{k}\right),
\end{aligned}
$$

Where $N$ is the number of grid points in the $x$-direction, $c_{i k}^{(1)}$ and $c_{i k}^{(n)}$ are the weighting coefficient associated with the first and $n$ th-order derivative, respectively, and $w\left(x_{k}\right)$ are field variables at the point $x_{k}(k=1,2, \ldots, M)$.

Weighting coefficients for the first and $n$ th-order derivative are obtained from the following recurrence equations,
Ali Nikkhoo et al. Indian J.Sci.Technol. 


$$
\begin{aligned}
& c_{i k}^{(1)}=\frac{R^{(1)}\left(x_{i}\right)}{\left(x_{i}-x_{k}\right) R^{(1)}\left(x_{k}\right)} \quad i \neq k, \quad i, k=1,2, \ldots, N, \\
& c_{i k}^{(n)}=n\left(c_{i i}^{(n-1)} c_{i k}^{(1)}-\frac{c_{i k}^{(n-1)}}{x_{i}-x_{k}}\right) \quad i \neq k, \\
& n=2,3, \ldots, N-1, \quad i, k=1,2, \ldots, N, \\
& c_{i i}^{(n)}=-\sum_{k=1, k \neq i}^{N} c_{i k}^{(n)} \quad n=1,2, \ldots, N-1, i=1,2, \ldots, N,
\end{aligned}
$$

Where $R(x)$ and $R^{(1)}(x)$ are defined as:

$$
\begin{aligned}
& R(x)=\left(x-x_{1}\right) \cdot\left(x-x_{2}\right) \cdots\left(x-x_{N}\right), \\
& R^{(1)}\left(x_{i}\right)=\prod_{k=1, k \neq i}^{N}\left(x_{i}-x_{k}\right) .
\end{aligned}
$$

$x_{1}, x_{2}, \ldots, x_{N}$ are coordinates of the grid points that might be selected as desired. Obviously, weighting coefficients of the second and higher-order derivatives is computable via weighting coefficients of the first-order derivative (Eqs. (3-5)). It has been proved that multi-dimensional cases, similar to one-dimensional case, are computable separately in any directions (Shu, 1991).

\section{Curved beam equations excited by a moving load}

The beam is assumed as a plane circular arch of constant cross section and an incompressible centerline. It is essential to utilize the polar coordinates with the origin at the center of the arch, and denotes by $r$ the radius of the arch, $\varphi$ the variable angle, $s$ the arch length equal to $\varphi r$ and $\mathrm{d} s$ the variations along the arch equal to $r \mathrm{~d} \varphi$. One element is taken out of the arch (Fig. 1) and the element equilibrium conditions in the tangential and normal direction as well as the moment conditions are written.

Neglecting the effect of shear, element rotation and infinitely small quantities of higher orders, the following equations would be obtained

Fig. 1.Equilibrium of an element of circular centerline beam

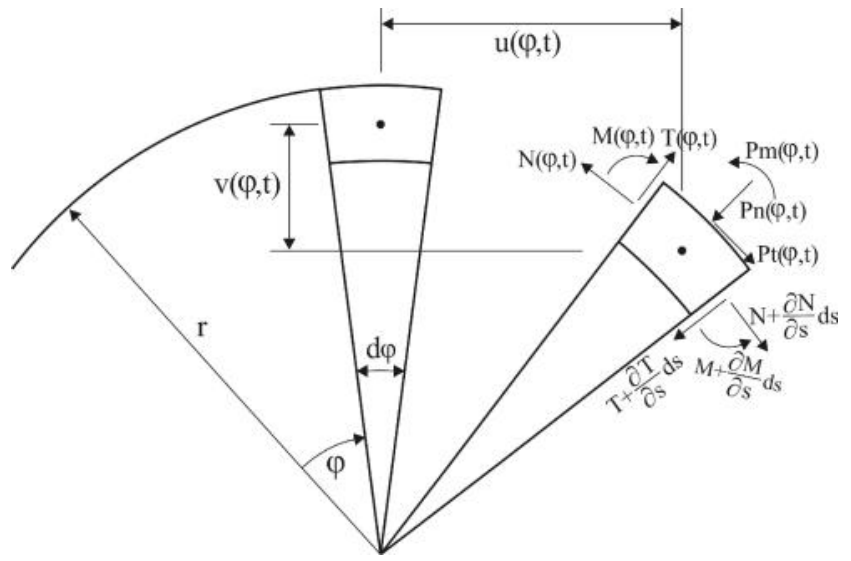

$$
\begin{aligned}
& \frac{\partial T(\varphi, t)}{\partial \varphi}+N(\varphi, t)-\mu r \frac{\partial^{2} v(\varphi, t)}{\partial t^{2}}+r p_{n}(\varphi, t)=0, \\
& \frac{\partial N(\varphi, t)}{\partial \varphi}-T(\varphi, t)-\mu r \frac{\partial^{2} u(\varphi, t)}{\partial t^{2}}+r p_{t}(\varphi, t)=0, \\
& \frac{\partial M(\varphi, t)}{\partial \varphi}-r T(\varphi, t)+r p_{m}(\varphi, t)=0,
\end{aligned}
$$

Where $u(\varphi, t)$ represents tangential displacement, $v(\varphi, t)$ radial displacement, $T(\varphi, t)$ shear force, $N(\varphi, t)$ normal force, $M(\varphi, t)$ bending moment, $p_{n}(\varphi, t)$ normal load per unit length of arch, $p_{t}(\varphi, t)$ tangential load per unit length of arch, $p_{m}(\varphi, t)$ bending load per unit length of arch and $\mu$ constant mass per unit of length arch. Further, for arches with incompressible centerlines and large radial of curvature:

$$
\begin{aligned}
& M(\varphi, t)=-\frac{E I}{r^{2}}\left[\frac{\partial^{2} v(\varphi, t)}{\partial \varphi^{2}}+\frac{\partial u(\varphi, t)}{\partial \varphi}\right], \\
& v(\varphi, t)=\frac{\partial u(\varphi, t)}{\partial \varphi},
\end{aligned}
$$

Where $E$ is the Young's modulus and / is moment of inertia at the beam sections.

By eliminating all unknowns (Eqs. (8-12) except $u(\varphi, t)$, the following single partial differential equation is obtained (Fryba, 1999).

$$
\begin{aligned}
& \frac{\partial^{6} u(\varphi, t)}{\partial \varphi^{6}}+2 \frac{\partial^{4} u(\varphi, t)}{\partial \varphi^{4}}+\frac{\partial^{2} u(\varphi, t)}{\partial \varphi^{2}} \\
& +\frac{\mu r^{4}}{E I}\left[\frac{\partial^{4} u(\varphi, t)}{\partial \varphi^{2} \partial t^{2}}-\frac{\partial^{2} u(\varphi, t)}{\partial t^{2}}\right] \\
& =\frac{r^{3}}{E I}\left[r \frac{\partial p_{n}(\varphi, t)}{\partial \varphi}-r p_{t}(\varphi, t)+\frac{\partial^{2} p_{m}(\varphi, t)}{\partial \varphi^{2}}+p_{m}(\varphi, t)\right]
\end{aligned}
$$

Taking the solution of this non-homogeneous equation, though possible, would be very time-consuming and laborious. Therefore, the approximate estimations as numerical methods are required to be employed.

\section{Problem formulation via DQM}

In this section, DQM application for analyzing an Euler-Bernoulli curved beam deformation under a moving load will be investigated. Consider a hinged-hinged beam as one with an arched shape, central angle $\phi$, radius $r$ and symmetric about the central axis with the hinges at equal heights (Fig. 2a). A vertical load, $P(x, t)$, moves horizontally over the arch at the constant speed, $c$. The load components in polar coordinate are
Research article (CIndian Society for Education and Environment (iSee) 


$$
\begin{aligned}
& p_{n}(\varphi, t)=p(x, t) \cos (\varphi-\phi / 2), \\
& p_{t}(\varphi, t)=p(x, t) \sin (\varphi-\phi / 2), \\
& p_{m}(\varphi, t)=0
\end{aligned}
$$

\section{Fig.2. Circular arch a) motion of a vertical force} b) bending vibration c) radial vibration

a)

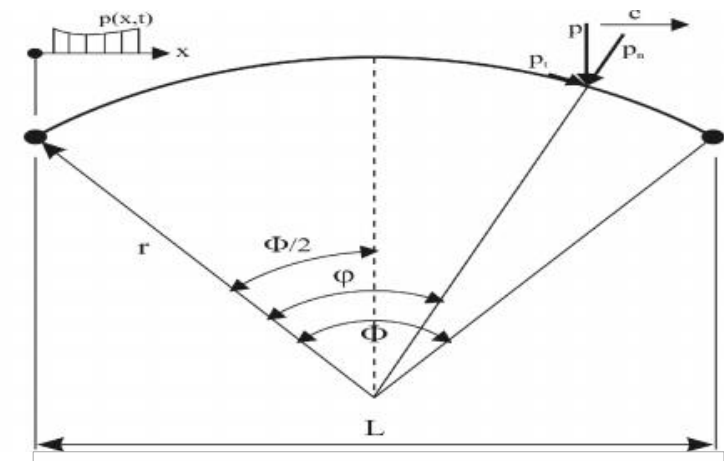

b)

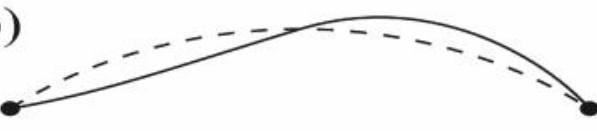

c)

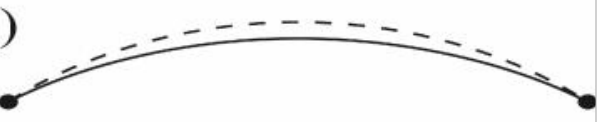

As it is known, in a hinged-hinged arches one differentiates between bending vibration (Fig. 2b) and radial vibration (Fig. 2c). Usually, the bending vibration that has the lowest natural frequency is taken as the dominant vibrational mode for solution. Radial vibration does not satisfy the assumption concerning the incompressibility of the centerline. However, in largespan, thin, curved bars the elongation of the centerline is very small in comparison with the effect of bending (Fryba, 1999).

In order to solve the differential equation of a curved beam deformation, Eq. (13), time equations can be solved as independent parameters and by considering initial conditions of the problem. Consequently, Eq. (13) has been transformed into a one-dimensional equation. But, in this paper, time was also considered as an independent parameter and Eq. (13) was analyzed via $D Q$ method as a partial differential equation in two dimensions of time and space. Expanding Eqs. (1-7) in directions of time and displacement and in polar coordinates is as follows:

$$
\begin{aligned}
& \frac{d^{n} w\left(\varphi_{i}, t_{j}\right)}{d \varphi^{n}}=\sum_{k=1}^{N} c_{i k}^{(n)} \cdot w\left(\varphi_{k}, t_{j}\right) \\
& i=1,2, \ldots, N, \quad j=1,2, \ldots, M,
\end{aligned}
$$

$$
\begin{aligned}
& \frac{d^{m} w\left(\varphi_{i}, t_{j}\right)}{d t^{m}}=\sum_{k=1}^{M} \bar{c}_{j k}^{(m)} \cdot w\left(\varphi_{i}, t_{k}\right) \\
& i=1,2, \ldots, N, \quad j=1,2, \ldots, M,
\end{aligned}
$$

Eq. (15) represents the value of the $n$ th-order derivative for $w(\varphi, t)$ function in the th grid point based on $\varphi$ (angle variation) while Eq. (16) represents the value of $m$ th-order for $w(\varphi, t)$ function in the th grid point based on $t$ (time). In these equations, $N$ corresponds to the number of grid points along $\varphi, M$ the number of grid points in $t$ domain,

Fig. 3 Normalized maximum tangential displacement via Galerkin method, FEM and DQM

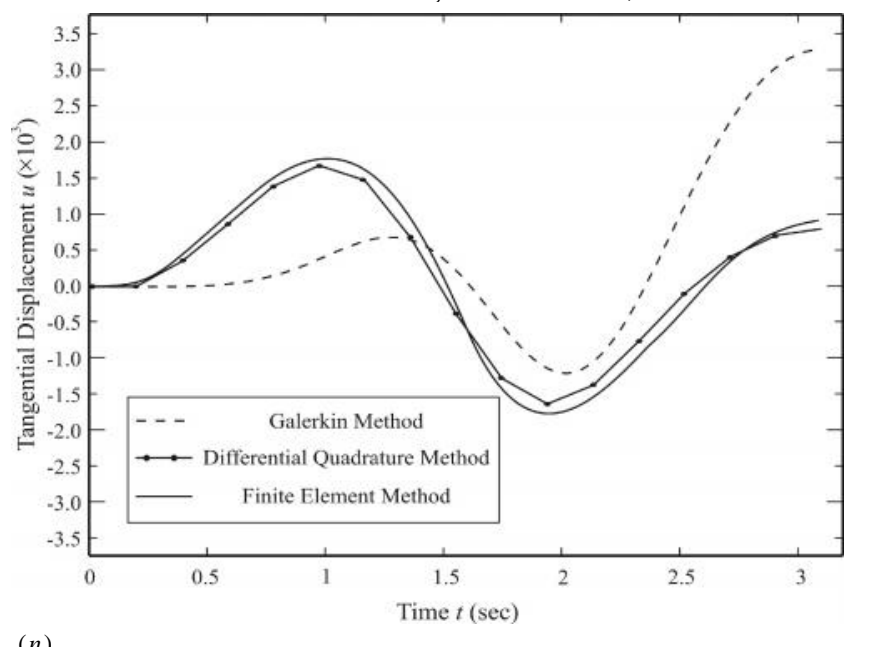

$c_{i k}^{(n)}$ weighting coefficients for the $n$ th-order derivative along $\varphi, \bar{c}_{j k}^{(m)}$ weighting coefficients for the $m$ th-order derivative in $t$ domain and $w\left(\varphi_{i}, t_{j}\right)$ function value in $i$ th grid point. Weighting coefficients for the first-order derivative along $\varphi$, first-order derivative in $t$ domain, $n$ th-order derivative along $\varphi$ and $m$ th-order derivative in $t$ domain are obtained

$c_{i j}^{(1)}=\frac{R^{(1)}\left(\varphi_{i}\right)}{\left(\varphi_{i}-\varphi_{j}\right) R^{(1)}\left(\varphi_{j}\right)} \quad i \neq j, i, j=1,2, \ldots, N$,

$c_{i j}^{(n)}=n\left(c_{i i}^{(n-1)} c_{i j}^{(1)}-\frac{c_{i j}^{(n-1)}}{\varphi_{i}-\varphi_{j}}\right)$

$i \neq j, n=2,3, \ldots, N-1, i, j=1,2, \ldots, N$,

$c_{i i}^{(n)}=-\sum_{j=1, j \neq i}^{N} c_{i j}^{(n)} n=1,2, \ldots, N-1, i=1,2, \ldots, N$

$\bar{c}_{i j}^{(1)}=\frac{Q^{(1)}\left(t_{i}\right)}{\left(t_{i}-t_{j}\right) Q^{(1)}\left(t_{j}\right)} \quad i \neq j, \quad i, j=1,2, \ldots, M$, 


$$
\begin{aligned}
& \bar{c}_{i j}^{(m)}=m\left(\bar{c}_{i i}^{(m-1)} \bar{c}_{i j}^{(1)}-\frac{\bar{c}_{i j}^{(m-1)}}{t_{i}-t_{j}}\right) \\
& i \neq j, m=2,3, \ldots, M-1, \quad i, j=1,2, \ldots, M, \\
& \bar{c}_{i i}^{(m)}=-\sum_{j=1, j \neq i}^{M} \bar{c}_{i j}^{(m)} \\
& m=1,2, \ldots, M-1, \quad i=1,2, \ldots, M,
\end{aligned}
$$

Where $R^{(1)}\left(\varphi_{k}\right)$ and $Q^{(1)}\left(t_{k}\right)$ are defined as

$$
\begin{aligned}
& R^{(1)}\left(\varphi_{k}\right)=\prod_{j=1, j \neq k}^{N}\left(\varphi_{k}-\varphi_{j}\right), \\
& Q^{(1)}\left(t_{k}\right)=\prod_{j=1, j \neq k}^{M}\left(t_{k}-t_{j}\right) .
\end{aligned}
$$

$\varphi_{i}$ represents the / th grid point coordinate, which would be selected as desired. $t_{j}$ corresponds to the $j$ th moment that proceeds in $t$ domain. Chebyshev grid point distribution model is chosen as the grid point distribution model along the domain since it corresponds well with the interpolation of polynomials:

$$
\begin{gathered}
\varphi_{i}=\frac{1-\cos \left(\frac{i-1}{N-1} \pi\right)}{2}, \quad i=1,2, \ldots N, \\
t_{j}=\frac{1-\cos \left(\frac{j-1}{M-1} \pi\right)}{2}, \quad j=1,2, \ldots M,
\end{gathered}
$$

\section{Numerical Examples}

Eq. (13) would be solved via DQM for the SS-SS curved beam. The curved beam was assumed as a semicircular arch with the opening angle of the arch $\phi=\pi$ radian, radius $r=1 \mathrm{~m}$, moving load with constant value of $P=1 \mathrm{~N}$ with constant speed of $c=1 \mathrm{~m} / \mathrm{s}$, Young's modulus of $E=1 \mathrm{~N} / \mathrm{m}$, moment of inertia $l=1 \mathrm{~m}^{4}$ and mass per unit of length $\mu=1 \mathrm{~kg} / \mathrm{m}$. The boundary conditions were applied separately based on Eq. (27) corresponding to the SS-SS boundary conditions and $u(\varphi, 0)=0$ and $\partial u(\varphi, 0) / \partial t=0$ as the initial conditions.

$$
\begin{aligned}
& u(0, t)=0, \quad u(\Phi, t)=0, \\
& v(0, t)=0, \quad v(\Phi, t)=0, \\
& M(0, t)=0, \quad M(\Phi, t)=0,
\end{aligned}
$$

In these equations, bending moment $(M)$ is obtained via Eq. (11) and radial displacement ( $v$ ) via Eq. (12). In order to apply boundary and initial conditions separately, the Generalized Differential Quadrature Method was utilized since it provides acceptable convergence in obtaining
Table 1. The numerical analysis results for curved beam via $D Q$

\begin{tabular}{|c|c|c|c|}
\hline \multicolumn{5}{c}{ method } \\
\hline Number of grid points & DAF $_{1}$ & DAF $_{2}$ & DAF $_{3}$ \\
\hline 11 & 3.326204 & 1108.735 & 2498.765 \\
\hline 15 & 2.277727 & 759.2423 & 1711.111 \\
\hline 19 & 2.243216 & 747.7386 & 1685.185 \\
\hline
\end{tabular}

analysis results of high-order differential equations (Wang et al., 2005).

The results are normalized. The tangential displacements, radial displacements and bending moments of the numerical solutions for the curved beam under a moving load are divided by the tangential displacement, radial displacement and bending moment of the analytical solution same prismatic circular arch, subjected to a concentrated unit force, $P$, at the middle of the beam, respectively. The analytical solution can be obtained by using the complementary virtual work method to solve this statically indeterminate structural problem (Tsumura, 1956).

The results of the mentioned beams dynamic

Table 2. The normalized maximum tangential displacements analyzed via DQM, FEM and Galerkin method

\begin{tabular}{|c|c|c|c|}
\hline $\begin{array}{c}\text { Number of } \\
\text { grid points }\end{array}$ & DQM & FEM & $\begin{array}{c}\text { Galerkin Method } \\
\text { (Fryba, 1999) }\end{array}$ \\
\hline 11 & 2498.765 & 2298.765 & 3351.852 \\
\hline 15 & 1711.111 & 2002.469 & 3351.852 \\
\hline 19 & 1685.185 & 1743.21 & 3351.852 \\
\hline
\end{tabular}

analysis solved via DQM are indicated in Table 1 for 11 , 15, and 19 nodes along $\varphi$ and in $t$ domain. The Dynamic Amplification factors for the normalized maximum bending moments $\left(\mathrm{DAF}_{1}\right)$, maximum tangential displacements $\left(\mathrm{DAF}_{2}\right)$ and maximum radial displacements $\left(\mathrm{DAF}_{3}\right)$ are assumed.

Table 2 shows the curved beam normalized maximum tangential displacement results under a moving load excitation passing at $1 \mathrm{~m} / \mathrm{s}$ constant speed via DQM, Finite Element Method, and Galerkin method. In the Finite Element solution, three-node elements were utilized while 11,15 , and 19 elements were respectively employed. Given that each element includes three degrees of freedom (DOF), the sum of the DOFs along the beam in FEM analysis exceeds to that of DQM.

Fig. 3 demonstrates the curved beam normalized tangential displacement corresponding to SS-SS boundary conditions traversed by a moving load via before mentioned methods.

Galerkin solution, which is only applicable for an SSSS beam, assumes a large curvature radius and a flat arch for the curved beam. This assumption is feasible when the central angle is taken small which in this circumstance; the load components are removed from the sinusoidal form. Consequently, in the semi-circular curved beam with the central angle $\Pi$, the results of Galerkin method, the only method offering closed-form solution for the curved beam problems under a moving 
Fig.4. Convergence of the maximum radial displacement of an SS-SS curved beam

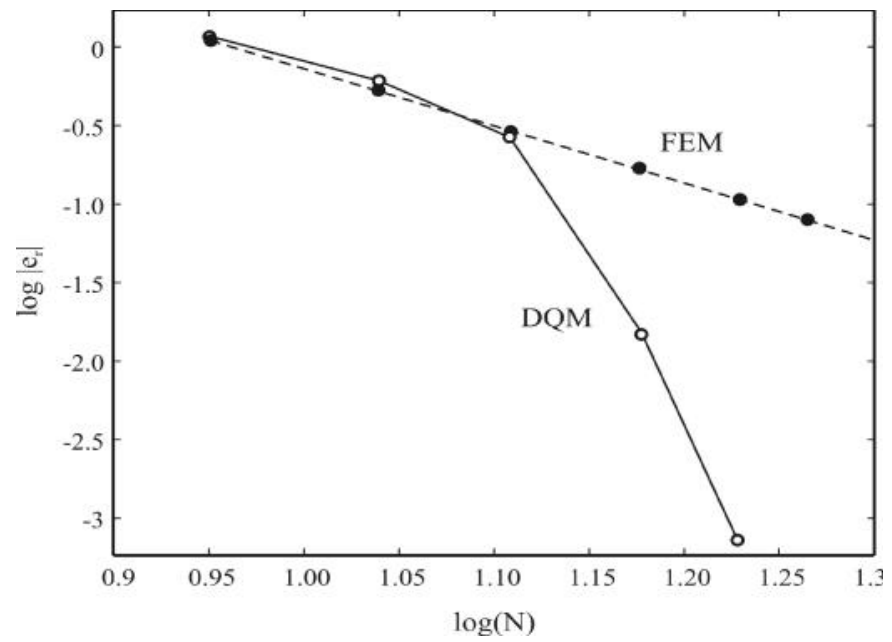

load, show an appreciable difference in results obtained by those employed numerical methods.

In Fig. 4 the DQM convergence rate has been compared to the FEM. The rate of convergence for the DQM increased when the number of grid points increases. The FEM convergence is constant by increasing the number of elements.

\section{Conclusions}

Considering the mentioned issues and the curved beam dynamic analysis excited by a moving load, it would be concluded that:

- The numerical examples revealed DQM provides acceptable convergence in solving high-order differential equations compared to other numerical methods. DQ method was assessed in the dynamic inplane deflection analysis model of prismatic curved beams, and it was illustrated that this numerical method is adequately efficient in these problems.

- In DQM, convergence rate augments quadratically as the number of grid points increases and almost complete convergence would be achieved by assuming 20 grid points in the whole domain. However, when the number of grid points exceeds a certain limit, DQM leads to an ill-conditioned problem. In the FEM, convergence rate is constant.

- Initial conditions, rigid boundary conditions and boundary conditions of the displacement field derivative were applied simultaneously in the start/end grid points of the domain. It was revealed that DQM provides suitable compatibility with applying initial conditions, rigid boundary conditions and bending moments.

- Application of the Galerkin method which generates a closed-form solution for curved beams problems excited by a moving load has some restrictions according to the geometry and boundary condition of the structure. If the curved beam is assumed to be semicircular curved one, the results obtained by this method show remarkable errors. Consequently, numerical methods must be utilized in order to have accurate analyses in such problems.

- DQ method is of relative superiority compared to the other numerical methods given its independence from domain discretization in the structural problems with particular geometry.

\section{References}

1. Bellman $R$ and Casti $\mathrm{J}$ (1971) Differential quadrature and long-term integration. J. Maths. Analysis \&Appli. 34, 235-238.

2. Bellman R, Kashef BG and Casti J (1972) Differential quadrature: a technique for the rapid solution of nonlinear partial differential equations. $J$. Computational Phys. 10, 40-52.

3. Bert CW, Jang SK and Striz AG (1988) Two new approximate methods for analyzing free vibration of structural components. AIAA J. 26, 612-618.

4. Fryba $L$ (1999) Vibration of solids and structures under moving loads. Thomas Telford, London.

5. Jang SK, Bert CW and Striz AG (1989) Application of differential quadrature to deflection and buckling of structural components. Intl. J. Numerical Methods Engg. 28, 561-577.

6. Laura P.A.A and Gutierrez R.H (1993) Analysis of vibrating Timoshenko beams using the method of differential quadrature. Shock \& Vibration. 1, 89-93.

7. Liu G.R and Wu T.Y (2001) Vibration analysis of beams using the generalized differential quadrature rule and domain decomposition. J. Sound \& Vibration. 246, 461-481.

8. Shu C (2000) Differential Quadrature and its application in engineering. Springer. London.

9. Shu C (1991) Generalized differential-integral quadrature and application to the simulation of incompressible viscous flows including parallel computation. PhD thesis. Univ. Glasgow, Scotland.

10.Tsumura $T$ (1956) Strength Design Data Book. Shokabo Publ. Co. Ltd. Tokyo.

11.Wang X, Liu F, Wang X and Gan L (2005) New approaches in application of differential quadrature method to fourth-order differential equations. Commun. Numerical Methods in Engg. 21, 61-71. 